\title{
ESTUDO DO DESEMPENHO DOS DIFERENTES SISTEMAS PROPULSIVOS PARA A TRANSFERÊNCIA DE UM SATÉLI- TE PARA UMA ÓRBITA GEOESTACIONÁRIA
}

\author{
FÁBIO A. S. MOTA ${ }^{1}$, JOSÉ N. HINCKEL ${ }^{2}$. \\ ${ }^{1}$ Instituto Nacional de Pesquisas Espaciais, São José dos Campos, Brasil, biomota@yahoo.com.br \\ ${ }^{2}$ Instituto Nacional de Pesquisas Espaciais, São José dos Campos, Brasil, hinckeljn@gmail.com
}

\begin{abstract}
Resumo— Neste trabalho é feito o estudo da injeção de um satélite em uma órbita geoestacionária. Foi considerado a injeção a partir de uma órbita baixa circular, com diferentes inclinações em relação ao plano do Equador. Para a transferência de órbita foi estudado a transferência de Hohmann, a transferência de Hohmann com mudança de plano e a transferência por propulsão elétrica, onde foi usada a equação de Edelbaum para estimar o $\Delta V$ mínimo da manobra. Com a estimação do $\Delta V$, foi analisado o consumo de vários tipos de propelentes. Os propulsores elétricos apresentaram consumo de propelente consideravelmente menor em relação aos propulsores químicos, no entanto, o tempo de transferência orbital dos propulsores elétricos é significativamente maior.
\end{abstract}

Palavras-chave—Manobra orbital, geoestacionária, propulsão.

\section{Introdução}

Os satélites artificiais descrevem as mais diversas órbitas, no entanto, a órbita geoestacionária é uma das mais importantes. Uma órbita geoestacionária, conhecida comumente pelo acrônimo GEO (do inglês Geostationary Earth Orbit), é uma órbita circular sobre o plano do Equador da Terra a $35786 \mathrm{~km}$ de altitude. Um satélite posicionado nessa órbita gira na mesma direção e possui a mesma velocidade angular da Terra, portanto, se encontra parado relativamente a um ponto fixo sobre a Terra. Atualmente 435 dos 1071 satélites operacionais $(41 \%)$ são geoestacionários (Technical Issues: UCS Satellite Database, 2013).

A injeção de um satélite em órbita se faz normalmente por meio de um foguete multiestágio. Existem basicamente dois tipos de lançamentos, por ascensão direta e através de uma órbita de espera. A órbita de espera é uma órbita baixa (órbitas baixas são comumente designadas por LEO, do inglês Low Earth Orbit) temporária, que tem altitude de aproximadamente $200 \mathrm{~km}$, ficando logo acima das camadas mais densas da atmosfera (Cornelisse et al., 1979). Existem várias razões para usar uma órbita de espera, entre elas podemos citar o aumento da janela de lançamento e missões para órbita geoestacionária. Para a transferência para uma órbita geoestacionária, o ideal seria que a órbita de espera ficasse no plano do Equador, resultando em menor consumo de propelente. No entanto, a inclinação da órbita de espera, $i$, depende da latitude de lançamento, $\delta$, e do azimute, A, conforme a equação (Tewari, 2007)

$$
\cos i=\cos \delta \sin A
$$

Da Eq. 1 fica evidente que a menor inclinação possível da órbita é alcançada quando o foguete é lançado na direção leste, ou seja, quando $A=90^{\circ}$. Assim, a inclinação da órbita nunca será menor que a latitude de lançamento. O sistema de propulsão encarregado de transferir o satélite da orbita de espera para a órbita de transferência é geralmente o último estágio do veículo lançador. Podemos classificar os métodos de transferência de órbita em três categorias:

\subsection{Transferência de Hohmann}

Normalmente se está interessado em transferir o satélite com o menor consumo de energia, onde geralmente a transferência de Hohmann é o método adotado. Neste caso, logo depois do veículo ser injetado na órbita de espera ou depois de um período orbitando, o veículo é injetado numa órbita elíptica de transferência, que tem seu perigeu tangenciando a órbita de espera e o apogeu tangenciando a órbita requerida. Esta é uma manobra bi-impulsiva, o motor é ligado primeiramente para transferir o veículo da órbita de espera para a órbita de transferência. Quando o veículo atinge o apogeu da órbita de transferência, que coincide com o raio da orbita final, o motor é acionado novamente para fazer a circularização da órbita (ver Figura 1.2a).

\subsection{Propulsão Química de Baixo Empuxo}

Para fazer uma transferência com propulsor químico de baixo empuxo, o satélite aplica uma série de empuxos sobre o perigeu, até o apogeu da órbita de transferência atingir o raio da órbita final. No apogeu, o propulsor é acionado mais algumas vezes até a circularização da órbita. Neste caso, como os propulsores são ligados sempre nas proximidades do perigeu e do apogeu, a eficiência total se aproxima a uma transferência de Hohmann (Wertz e Larson,1999) (ver Figura 1.2b).

\subsection{Propulsão Elétrica}

Este é o tipo de menor empuxo e, consequentemente, o que leva mais tempo para alcançar a órbita final, podendo durar meses. Conforme Figura 1.2c, podemos ver que a trajetória de transferência é em formato de espiral, diferente das manobras impulsivas. 


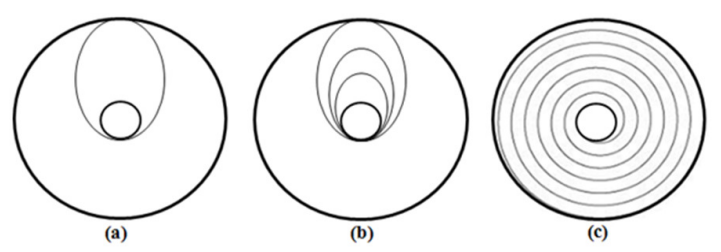

Figura 1. Desenho esquemático das manobras de transferência. (a) Transferência de Hohmann, (b) Transferência por propulsão química de baixo empuxo e (c) Transferência por propulsão elétrica.

\section{Propósito}

Este trabalho apresenta um estudo comparativo de diferentes sistemas propulsivos para a transferência de um satélite de uma órbita LEO de espera em diferentes inclinações para uma órbita GEO. O consumo de propelente e o tempo da transferência do satélite são analisados para as manobras impulsivas (propulsão química) e manobras de baixo empuxo (propulsão elétrica).

\section{Métodos}

Esta seção aborda as manobras de transferência de uma órbita circular de espera para a órbita geoestacionária utilizando o mínimo incremento de velocidade $\Delta V$, que é equivalente ao consumo de energia. Nas subseções seguintes são consideradas as transferências utilizando propulsores químicos, onde são consideradas manobras impulsivas (ou seja, é considerado que $\Delta V$ seja aplicado instantaneamente), e propulsores elétricos, que realizam manobras de baixo empuxo. Chobotov (1996) foi utilizado para o desenvolvimento das equações.

\subsection{Transferência de Hohmann}

A transferência de Hohmann é uma transferência biimpulsiva entre órbitas circulares coplanares com o menor consumo de propelentes, que é equivalente a uma transferência com o mínimo $\Delta V$.

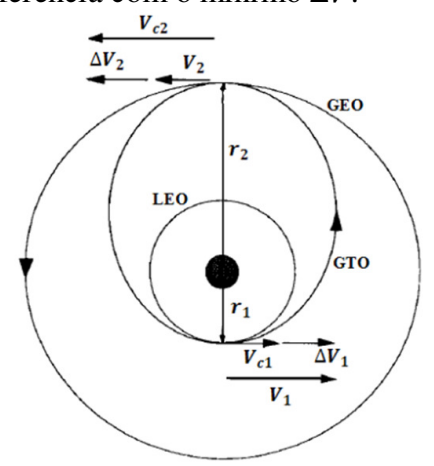

Figura 2. Transferência de Hohmann de LEO para GEO.

Para analisar a transferência de órbita de um satélite por meio de uma variação de velocidade, a equação da energia ou equação da "vis-visa" (Eq. 2) deve ser inicialmente considerada.

$$
V^{2}=\mu\left(\frac{2}{r}-\frac{1}{a}\right)
$$

onde Vé a magnitude da velocidade orbital em determinado ponto, $r$ é a distância desse ponto até o foco, $a$ é semieixo maior da órbita e $\mu$ é a constante geo-gravitacional. Usando a equação da "vis-viva" e a Figura 2, temos

$$
V_{c 1}=\sqrt{\frac{\mu}{r_{1}}} \quad V_{1}=\sqrt{\mu\left(\frac{2}{r_{1}}-\frac{2}{r_{1}+r_{2}}\right)}
$$

onde $V_{c 1}$ é a velocidade orbital da órbita circular inicial, $V_{1}$ é a velocidade de perigeu da órbita uma elíptica de transferência. O incremento total de velocidade para ir da órbita inicial para órbita de transferência é então

$$
\Delta V_{1}=V_{1}-V_{c 1}
$$

De maneira similar, o incremento de velocidade no apogeu para circularização da órbita elíptica de transferência é

$\Delta V_{2}=V_{c 2}-V_{2}=\sqrt{\frac{\mu}{r_{2}}}-\sqrt{\mu\left(\frac{2}{r_{2}}-\frac{2}{r_{1}+r_{2}}\right)}$

A variação de velocidade total para a manobra é então

$$
\Delta V_{T}=\Delta V_{1}+\Delta V_{2}
$$

O tempo de transferência da manobra é o dado pela metade do período da órbita elíptica de transferência

$$
T=\frac{P_{G T O}}{2}
$$

\subsection{Transferência de Hohmann com Mudança de Plano}

A transferência de Hohmann com mudança de plano é uma transferência ótima bi-impulsiva entre órbitas circulares de diferentes raios não coplanares (plano das órbitas faz um ângulo $\theta$ ). $\mathrm{O}$ primeiro $\Delta V$ não somente produz uma órbita elíptica de transferência com apogeu tangenciando a órbita final, mas também rotaciona o plano da órbita em um ângulo $\alpha_{1}$. No apogeu, o segundo $\Delta V$ circulariza e rotaciona o plano da orbita em um ângulo $\alpha_{2}$, onde $\theta=\alpha_{1}+\alpha_{2}$. Abaixo segue as equações e um esboço dos triângulos de velocidades.
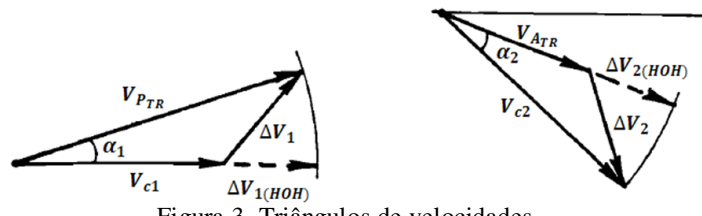

Figura 3. Triângulos de velocidades. 


$$
\begin{gathered}
\Delta V_{1}=\sqrt{V_{P_{T R}}^{2}+V_{c 1}^{2}-2 V_{P_{T R}} V_{c 1} \cos \alpha_{1}} \\
V_{P_{T R}}=V_{c 1}+\Delta V_{1(\mathrm{HOH})} \\
\Delta V_{2}=\sqrt{V_{A_{T R}}^{2}+V_{c 2}^{2}-2 V_{A_{T R}} V_{c 2} \cos \left(\theta-\alpha_{1}\right)} \\
V_{A_{T R}}=V_{c 2}-\Delta V_{2(\mathrm{HOH})} \\
\theta=\alpha_{1}+\alpha_{2}
\end{gathered}
$$

Para minimizar $\Delta V_{T}$, estabelecemos $\partial \Delta V_{T} / \partial \alpha_{1}=0$, ou seja

$$
\begin{aligned}
\frac{\partial \Delta V_{T}}{\partial \alpha_{1}} & =\frac{V_{P_{T R}} V_{c 1} \operatorname{sen} \alpha_{1}}{\Delta V_{1}} \\
& -\frac{V_{A_{T R}} V_{c 2} \operatorname{sen}\left(\theta-\alpha_{1}\right)}{\Delta V_{2}}=0
\end{aligned}
$$

Resolvendo iterativamente para $\alpha_{1_{O T}}$. Substituindo em $\Delta V_{1}$ e $\Delta V_{2}$, obtemos, respectivamente, $\Delta V_{1_{O T}} \mathrm{e}$ $\Delta V_{2_{O T}}$. Então

$$
\Delta V_{T_{M I N}}=\Delta V_{1_{O T}}+\Delta V_{2_{O T}}
$$

\subsection{Transferência de Baixo Empuxo}

Para a transferência com propulsão elétrica entre órbitas circulares inclinadas, será considerada a equação da trajetória ótima de Edelbaum para avaliar o $\Delta V$ da missão.

$$
\Delta V=\sqrt{V_{c 1}^{2}-2 V_{c 1}^{2} V_{c 2}^{2} \cos \left(\frac{\pi}{2} \Delta i\right)+V_{c 2}^{2}}
$$

onde $\Delta i$ é a variação da inclinação das órbitas. Foi assumido aceleração constante e ângulo do vetor de empuxo constante em cada revolução. Se $f$ é a aceleração do veículo, o tempo de transferência pode ser dado por

$$
T=\frac{\Delta V}{f}
$$

\subsection{Consumo de Propelente}

Estimado o $\Delta V$ necessário para realizar a transferência de órbita, o consumo de propelente pode ser calculado. Para isto, é introduzida a equação de Tsiolkovsky

$$
\Delta V=g_{0} I_{s p} \ln \frac{m_{i}}{m_{f}}
$$

onde $m_{i}$ é a massa inicial do veículo e $m_{f}$ é a massa final do veículo. Explicitando $m_{f}$ na Eq. 17, temos

$$
m_{f}=m_{i} e^{\left(-\Delta V / g_{0} I_{s p}\right)}
$$

Então a massa de propelente consumido é

$$
m_{p}=m_{i}\left[1-e^{\left(-\Delta V / g_{0} I_{s p}\right)}\right]
$$

\section{Resultados}

Para uma visualiação do consumo de propelente de um veículo espacial, na Figura 4 é apresentado a variação de consumo de propelente para uma transferência orbital com diferentes inclinações (ver Tabela 1 , onde $e$ é a ecentricidade, $i$ é a inclinação da órbita, $\Omega$ é a ascensão reta do nodo ascendente, $\omega$ é o argumento do perigeu e $M$ é a anomalia verdadeira). Importante destacar que geralmente é o último estágio do veículo lançador o responsável por aplicar o primeiro incremento de velocidade para transferir o veículo da órbita de espera para a órbita de transferência.

Tabela 1. Parâmetros para as órbitas inicial e final.

\begin{tabular}{|l|c|c|}
\hline Elemento orbital & LEO & GEO \\
\hline$a(\mathrm{~km})$ & 6578 & 42164 \\
\hline$e$ & 0 & 0 \\
\hline$i\left(^{\circ}\right)$ & $0-45^{\circ}$ & 0 \\
\hline$\Omega\left(^{\circ}\right)$ & 0 & 0 \\
\hline$\omega\left(^{\circ}\right)$ & N/A & N/A \\
\hline$M\left(^{\circ}\right)$ & livre & livre \\
\hline
\end{tabular}

Para os sistemas propulsivos químicos, foram considerados um híbrido e dois bipropelentes $\left(\mathrm{H}_{2} / \mathrm{O}_{2}\right.$ e $\mathrm{N}_{2} \mathrm{H}_{4} / \mathrm{N}_{2} \mathrm{O}_{4}$ ), onde o $\Delta V$ estimado foi para manobras impulsivas, ou seja, transferência de Hohmann. Os propulsores elétricos foram um SPT (do inglês Stationary Plasma Thruster) e um XIPS (do inglês Xenon Ion Propulsion System), onde foi considerada a equação de Edelbaum para estimação do $\Delta V$. 


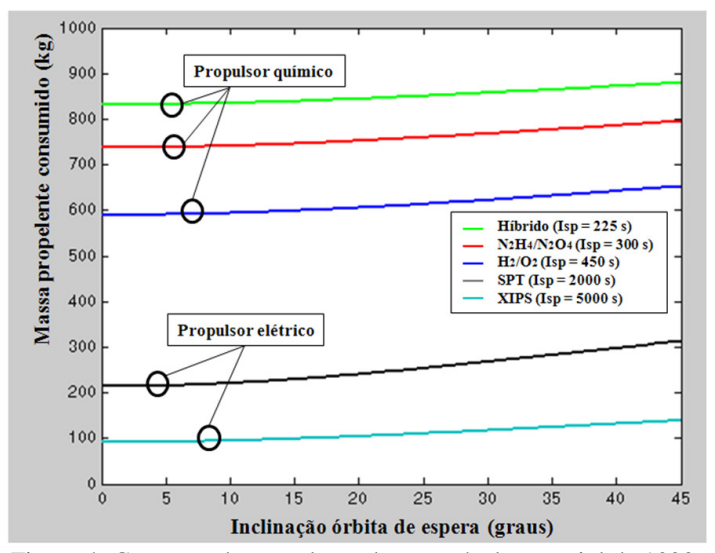

Figura 4. Consumo de propelente de um veículo espacial de 1000 $\mathrm{kg}$ de massa útil para injeção em uma órbita GEO a partir de uma órbita circular de espera de $200 \mathrm{~km}$ de altitude.

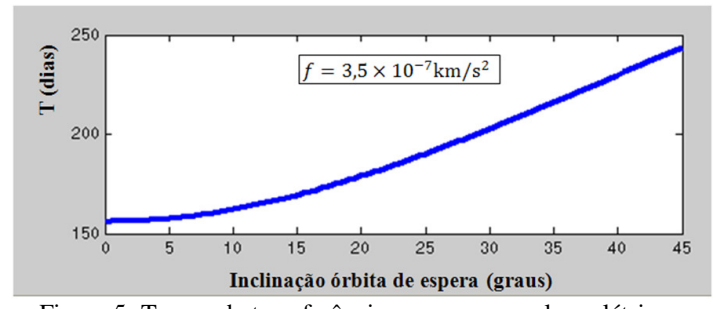

Figura 5. Tempo de transferência para um propulsor elétrico.

Da Figura 4, pode-se notar que o consumo de propelente é muito menor para os propulsores elétricos, pois apresentam $I_{s p}$ elevados. No entanto, enquanto uma manobra por transferência de Hohmann levaria metade do período da órbita de transferência, ou seja, 5,26 horas (No entanto para aplicações reais, o tempo de transferência pode chegar a semanas, pois a manobra geralmente é feita com mais de dois incrementos de velocidade), uma manobra com propulsão elétrica pode levar vários meses (Figura 5). Outro ponto que pode ser observado da Figura 4 é que a variação do consumo de propelente é relativamente pequena com a variação da inclinação entre as órbitas. Na Figura 6 é esboçado como o $\Delta V$ varia para uma transferência de uma órbita circular de $200 \mathrm{~km}$ de altitude para órbitas finais ciculares de maiores altitudes, considerando manobras impulsivas (propulsão química). Pode ser observado que o $\Delta V$ para uma órbita geoestacionária varia pouco com a inclinação da órbita LEO quando comparado a uma transferência para uma órbita final baixa, o que está de acordo com a Figura 4, onde o consumo de propelente foi relativamente pequeno com a variação da inclinação entre as órbitas.

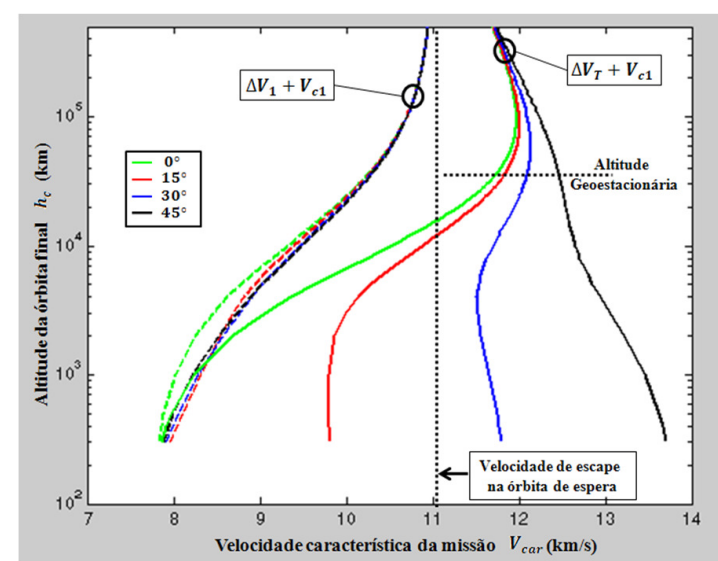

Figura 6. Velocidade característica para injeção em órbitas finais de altitudes crescentes considerando 4 órbitas iniciais de $200 \mathrm{~km}$ de altitude com diferentes inclinações usando transferência de

$$
\text { Hohmann. }
$$

Na Figura 6 também é mostrado que a medida que a altitude da órbita final cresce, o incremento de velocidade inicial mais a velocidade circular da órbita de espera $\left(\Delta V_{1}+V_{c 1}\right)$ tende a velocidade de escape da órbita. $\mathrm{O} \Delta V_{T}$ tende a ser pouco sensível com variações da inclinação dos planos orbitais a medida que a altitude da órbita final se torna muito grande, conforme pode ser observado na Figura 6, as curvas para diferentes inclinações tendem a convergir.

Como o tempo de transferência ainda é muito grande para a propulsão elétrica, seu uso operacional ainda se restringe a manobras de manutenção de órbitas. Enquanto limitações energéticas e de tecnologia de materiais tornam as manobras LEOGEO com propulsão elétrica proibitivas, métodos combinados usando propulsão química e elétrica tem sido objetivo de estudo. Johnson (2007), em sua tese de Mestrado, apresentou estratégias para uma injeção ótima em uma órbita GEO usando sistemas combinados de propulsão química e elétrica. Utilizando o programa SEPSPOT (Solar Eletric Propulsion Steering Program for Optimal Trajectory), o autor considerou 5 estratégias de inserção em órbita GEO, 3 utilizando sistemas combinados e 2 utilizando somente propulsão química ou elétrica. Estas duas últimas são comparadas com os métodos do presente artigo (Tabela 3).

Tabela 2. Capacidade de lançamento de carga útil de três foguetes americanos.

\begin{tabular}{|l|c|c|}
\hline Veículo Lançador & Payload GTO (kg) & Payload LEO (kg) \\
\hline Delta II (6925) & 1380 & 3850 \\
\hline Delta II (7925) & 1750 & 4900 \\
\hline Atlas 1 & 2200 & 5500 \\
\hline
\end{tabular}


Tabela 3. Comparação dos métodos.

\begin{tabular}{|l|c|c|c|}
\hline \multirow{2}{*}{ Lançador } & $\begin{array}{c}\text { Estratégia } \\
\text { Injeção } \\
\text { GEO }\end{array}$ & $\begin{array}{c}\mathbf{M}_{\text {f inal }}(\mathbf{k g}) \\
\text { (analítica) }\end{array}$ & $\begin{array}{c}\mathbf{M}_{\text {final }}(\mathbf{k g}) \\
\text { (SEPSPOT) }\end{array}$ \\
\hline \multirow{2}{*}{$\begin{array}{l}\text { Delta II } \\
\mathbf{6 9 9 2 5 )}\end{array}$} & $\begin{array}{c}\text { Química } \\
\text { (LV/GTO) }\end{array}$ & 807,7 & 807,9 \\
\cline { 2 - 4 } & $\begin{array}{c}\text { Elétrica } \\
\text { (LV/LEO) }\end{array}$ & 3237,3 & 3266,5 \\
\hline \multirow{2}{*}{$\begin{array}{c}\text { Delta II } \\
\mathbf{7 9 2 5 )}\end{array}$} & $\begin{array}{c}\text { Química } \\
\text { (LV/GTO) }\end{array}$ & 1024,2 & 1024,5 \\
\cline { 2 - 4 } & $\begin{array}{c}\text { Elétrica } \\
\text { (LV/LEO) }\end{array}$ & 4120,0 & 4138,2 \\
\hline \multirow{3}{*}{ Atlas 1 } & $\begin{array}{c}\text { Química } \\
\text { (LV/GTO) }\end{array}$ & 1287,6 & 1288,0 \\
\cline { 2 - 4 } & $\begin{array}{c}\text { Elétrica } \\
\text { (LV/LEO) }\end{array}$ & 4624,5 & 4640,2 \\
\hline
\end{tabular}

Na Tabela 2 são apresentadas as capacidades de lançamento de 3 foguetes americanos, onde os dados são usados na Tabela 3 para as 2 estratégias de lançamento. Uma estratégia considera que o último estágio do foguete lança o satélite para uma órbita GTO e o motor de apogeu do satélite completa a manobra, e a outra considera que o foguete lança o satélite até uma órbita LEO e o propulsor elétrico (XIPS) realiza a transferência. Para obtenção dos resultados foram considerados uma LEO de $300 \mathrm{~km}$ de altitude e inclinação de $28,5^{\circ}$, uma GTO com perigeu de $300 \mathrm{~km}$ e inclinação de $26,5^{\circ}$, propulsor químico com 340s de impulso específico e um XIPS com 3500s de impulso específico.

\section{Conclusão}

Neste trabalho foram abordadas as manobras de transferência de uma órbita de espera com diferentes inclinações para uma órbita GEO considerando manobras impulsivas (propulsão química) e manobras de baixo empuxo (propulsão elétrica). Com o $\Delta V$ estimado para as manobras de transferência, o consumo de propelentes pode ser comparado entre os sistemas propulsivos. Para as manobras impulsivas com mudança de plano, se constatou que quando a altitude da órbita final tende a valores muito grandes, o $\Delta V$ necessário para fazer a mudança de plano tende a ser desprezível, assim o $\Delta V$ total para a transferência orbital com mudança de plano tende ao $\Delta V$ calculado para transferência no plano.

$\mathrm{Na}$ propulsão elétrica, muito menos propelente foi necessário na transferência, no entanto, o tempo de transferência é muito maior. Assim a exposição do satélite no Cinturão de Van Allen é bem mais alta, podendo degradar os painéis solares reduzindo a vida útil da missão devido à exposição à radiação.

Por fim, foi feita uma comparação com os resultados de Johnson (2007), que utilizou um programa de otimização numérico chamado SEPSPOT, obtendo resultados em excelente concordância com a literatura.

\section{Agradecimentos}

À CAPES pela bolsa concedida.

\section{Referências}

Cornelisse, J. W.; Schöyer, H. F. R.; Wakker, K. F. (1979). Rocket Propulsion and Spaceflight Dynamics. London: Pitman. 505p.

Chobotov, V. A. (1996). Orbital Mechanics. Virginia: AIAA Education Series. 447p.

Johnson, D. W. (1997). Optimal Orbit Insertion Strategies Using Combined High and Low

Thrust Propulsion Systems. Master Thesis. Air Force Institute of technology.

Technical Issues: UCS Satellite Database (2013). Obtido de Union of Concerned Scientists: http://www.ucsusa.org.

Tewari, A. (2007). Atmospheric and Spaceflight Dynamics. Boston: Birkhäuser. 556p.

Wertz, J. R.; Larson, W. J. (1999). Space Mission Analysis and Design. Microcosm Press. 